\title{
Improved Power Flow Algorithm for VSC-HVDC System Based on High-Order Newton-Type Method
}

\author{
Yanfang Wei, ${ }^{1}$ Qiang He, ${ }^{2}$ Yonghui Sun, ${ }^{3}$ Yanzhou Sun, ${ }^{1}$ and Cong Ji ${ }^{4}$ \\ ${ }^{1}$ School of Electrical Engineering and Automation, Henan Polytechnic University, Jiaozuo 454000, China \\ ${ }^{2}$ Economics and Business College, Qingdao Technological University, Qingdao 266520, China \\ ${ }^{3}$ College of Energy and Electrical Engineering, Hohai University, Nanjing 210098, China \\ ${ }^{4}$ Jiangsu Frontier Electric Technology Co., Ltd., Nanjing 211102, China
}

Correspondence should be addressed to Yanfang Wei; weiyanfang@hpu.edu.cn

Received 4 April 2013; Accepted 12 May 2013

Academic Editor: Guanghui Wen

Copyright (C) 2013 Yanfang Wei et al. This is an open access article distributed under the Creative Commons Attribution License, which permits unrestricted use, distribution, and reproduction in any medium, provided the original work is properly cited.

\begin{abstract}
Voltage source converter (VSC) based high-voltage direct-current (HVDC) system is a new transmission technique, which has the most promising applications in the fields of power systems and power electronics. Considering the importance of power flow analysis of the VSC-HVDC system for its utilization and exploitation, the improved power flow algorithms for VSC-HVDC system based on third-order and sixth-order Newton-type method are presented. The steady power model of VSC-HVDC system is introduced firstly. Then the derivation solving formats of multivariable matrix for third-order and sixth-order Newton-type power flow method of VSC-HVDC system are given. The formats have the feature of third-order and sixth-order convergence based on Newton method. Further, based on the automatic differentiation technology and third-order Newton method, a new improved algorithm is given, which will help in improving the program development, computation efficiency, maintainability, and flexibility of the power flow. Simulations of AC/DC power systems in two-terminal, multi-terminal, and multi-infeed DC with VSC-HVDC are carried out for the modified IEEE bus systems, which show the effectiveness and practicality of the presented algorithms for VSC-HVDC system.
\end{abstract}

\section{Introduction}

Voltage source converter (VSC) based high-voltage directcurrent (HVDC) is a new technology of HVDC transmission system. Based on pulse width modulation and VSC, the VSC-HVDC system has many merits and attracted wide publicity worldwide [1-3]. Since the first pilot project application in 1997, the VSC-HVDC system is widely applied in interconnected power system, the connection of distributed generation to power grid, the supply of electric power to islands or offshore drilling platform, the distribution of power to urban power network, and so forth.

The main advantages of VSC-HVDC system are as follows: no synchronization problem of AC system, the feature of supplying power to passive network, the simultaneous and independent control for active power and reactive power, the easy achievement of inversion for power flow, the more flexible control modes, the suitable application for multi-terminal and multi-infeed system, and so on. In the near future, a series of new VSC-HVDC transmission system will be built and put into operation worldwide $[1,4]$. As a fundamental analytical method for operation and analyzing of power system, the reliable power flow calculation algorithm is an indispensable tool for AC/DC interconnected power systems [5-10]. A great deal of research has been conducted in this field. Now there are two critical methods for the power flow analysis of AC/DC systems, the unified iteration technique and the alternative iteration technique [10-14]. The former has the precision of quadratic convergence and has the better convergence for a variety of control modes of HVDC system. But the realization of the control modes switching for HVDC system is difficult. The latter has the trait of easy programming, especially for the varying process of HVDC control modes. But the alternative iteration technique is sensitive to the operation ways and control modes of the HVDC system and is inclined to the convergence problem. 
The Newton method is a fundamental and important technology to solve the power flow of power system [69, 15-17]. In [6], a Newton-Raphson power flow algorithm is proposed for the VSC-HVDC system. In [7], the steady power flow of VSC-HVDC is presented based on Newton method and alternative iteration technique. In [8], an optimal power flow (OPF) model suitable for VSC-HVDC system is presented based on Newton-Raphson algorithm. In [9], based on Newton method, a new model that considers the operational constraints associated with the MVA ratings of the converters for OPF analysis of VSC-HVDC system is introduced. In these papers, the modeling approach based on Newton algorithm only has the first-order or quadratic convergence, and the convergence precision needs to be further improved.

In recent years, the solution of nonlinear equation has made great progress, especially the modified Newton method with high-order convergence performance [18-23]. In [23], the power flow algorithm with cubic convergence is analyzed for power system. Despite the fact that a great many improved algorithms for power flow analysis of AC/DC interconnected networks with VSC-HVDC have been presented in many aspects, few papers use the Newton method of high-order convergence to analyze the power flow of VSC-HVDC system. Moreover, with the VSC-HVDC adding to AC/DC system, the kind and number of the system variables are multiplied, causing the modeling of AC/DC system to become more complex. And with the increase of DC line of VSCHVDC, the dimension and scale of Jacobian matrix and equations of AC/DC system are increased obviously, causing the efficiency of hand codes to decrease. The automatic differentiation (AD) technology overcomes the shortcomings of hand codes. Compared with other differential methods, such as numerical differentiation and symbolic differentiation methods, the $\mathrm{AD}$ has the advantages of no truncation error, the exact solution of Jacobian matrix, the less work of hand codes, and so on [24-26]. Motivated by the above discussions, we will investigate the problem of power flow for AC/DC systems with VSC-HVDC. Novel third-order and sixth-order convergence of power flow technique based on Newton method will be derived, and the AD technology will be introduced in this paper based on third-order Newton method to raise the efficiency of programming. The effectiveness of the presented arithmetics for VSC-HVDC system is also discussed. These results and observations will help promote the practical applications of high-order Newtontype method in AC/DC systems with VSC-HVDC.

The remaining of this paper is arranged as follows. In Section 2, a mathematical model for the VSC-HVDC system is presented, in which all the AC system equations, the VSC equations, and the control modes of VSC are analyzed basing on steady model. In Section 3, the power flow and converter equations of VSC-HVDC, the mathematical description of Newton method, third-order and sixth-order convergence of Newton-type methods, and the improved third-order Newton method based on automatic differentiation are presented. In Section 4, the methods are applied to the modified IEEE bus test systems with VSC-HVDC. This paper ends with a conclusion finally.

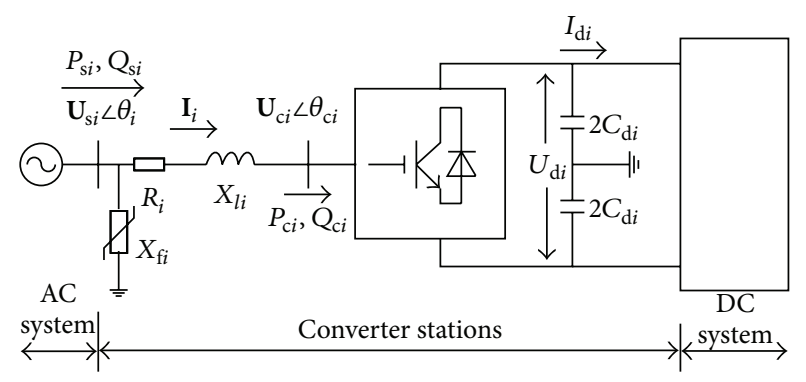

FIGURE 1: Schematic diagram of steady state physical model for multi-terminal VSC-HVDC.

\section{Mathematical Steady Model of VSC-HVDC System}

2.1. Per-Unit Value System of VSC-HVDC. For the simulation and calculation of AC/DC hybrid power systems, the unified per-unit value system should be adopted both for AC system and DC system. In this paper the per-unit value system is introduced as follows [7]:

$$
\begin{gathered}
P_{\mathrm{dB}}=S_{\mathrm{B}}, \\
U_{\mathrm{dB}}=U_{\mathrm{B}}, \\
I_{\mathrm{dB}}=\sqrt{3} I_{\mathrm{B}}, \\
R_{\mathrm{dB}}=Z_{\mathrm{B}},
\end{gathered}
$$

where $S_{\mathrm{B}}, P_{\mathrm{dB}}$ are the reference power of AC system and DC system, respectively. $U_{\mathrm{B}}, I_{\mathrm{B}}$, and $Z_{\mathrm{B}}$ are the reference voltage, reference current, and reference impedance of $A C$ side of the converter, respectively. $U_{\mathrm{dB}}, I_{\mathrm{dB}}$, and $R_{\mathrm{dB}}$ are the reference voltage, reference current, and reference impedance of DC side of the converter, respectively.

2.2. Mathematical Steady Model of VSC-HVDC System. The VSC-HVDC system consists of at least two VSC stations, one operating as a rectifier station and the other as an inverter station. The VSC stations can be connected as twoterminal, multi-terminal, or multi-infeed DC system with VSC-HVDC, depending on the various different applications fields [1-3]. The steady state physical model for a multiterminal DC system with VSC-HVDC is shown schematically in Figure 1. The steady models of VSC-HVDC are given in the per-unit system (p.u.) as follows:

$$
\begin{gathered}
\mathbf{I}_{i}=\frac{\mathbf{U}_{\mathrm{si}}-\mathbf{U}_{\mathrm{c} i}}{R_{i}+j X_{l i}}, \\
\widetilde{S_{s i}}=P_{\mathrm{s} i}+j Q_{\mathrm{si}}=\mathbf{U}_{\mathrm{si} i} \mathbf{I}_{i}^{*}, \\
P_{\mathrm{si}}=-\left|Y_{i}\right| U_{s i} U_{\mathrm{c} i} \cos \left(\delta_{i}+\alpha_{i}\right)+\left|Y_{i}\right| U_{s i}^{2} \cos \alpha_{i},
\end{gathered}
$$




$$
\begin{gathered}
Q_{s i}=-\left|Y_{i}\right| U_{s i} U_{c i} \sin \left(\delta_{i}+\alpha_{i}\right)+\left|Y_{i}\right| U_{s i}^{2} \sin \alpha_{i}+\frac{U_{s i}^{2}}{X_{\mathrm{f} i}}, \\
P_{\mathrm{c} i}=\left|Y_{i}\right| U_{s i} U_{c i} \cos \left(\delta_{i}-\alpha_{i}\right)-\left|Y_{i}\right| U_{c i}^{2} \cos \alpha_{i}, \\
Q_{\mathrm{c} i}=-\left|Y_{i}\right| U_{s i} U_{c i} \sin \left(\delta_{i}-\alpha_{i}\right)-\left|Y_{i}\right| U_{c i}^{2} \sin \alpha_{i}, \\
P_{\mathrm{d} i}=U_{\mathrm{d} i} I_{\mathrm{d} i}=\left|Y_{i}\right| U_{\mathrm{s} i} U_{\mathrm{c} i} \cos \left(\delta_{i}-\alpha_{i}\right)-\left|Y_{i}\right| U_{\mathrm{c} i}^{2} \cos \alpha_{i}, \\
U_{\mathrm{c} i}=\frac{\sqrt{6} M_{i} U_{\mathrm{d} i}}{4} .
\end{gathered}
$$

The variables in the equations of (2)-(9) are referenced to the literature [14].

2.3. Steady-State Control Modes of VSC-HVDC. Owning to having full controllable power electronic switch semiconductors such as insulated gate bipolar transistor and gate turn-off thyristor, VSC-HVDC has the ability to independent control active and reactive power at its terminal. So for each VSC, a couple of regular used control goals can be set [27].

(1) AC active power control: determines the active power exchanged with the AC system.

(2) DC voltage control: is used to keep the DC voltage control constant.

(3) AC reactive power control: determines the reactive power exchanged with the AC system.

(4) AC voltage control: instead of controlling reactive power, AC voltage can be directly controlled, determining the voltage of the system bus.

The general used control means of VSC include the following four categories:

(1) constant $\mathrm{DC}$ voltage control, constant $\mathrm{AC}$ reactive power control;

(2) constant DC voltage control, constant AC voltage control;

(3) constant AC active power control, constant AC reactive power control;

(4) constant $\mathrm{AC}$ active power control, constant $\mathrm{AC}$ voltage control.

\section{The Improved Power Flow Algorithms of AC/DC Systems with VSC-HVDC Based on High-Order Newton-Type Method}

3.1. Steady Mathematical Model of Power Flow Calculation of VSC-HVDC System. For the AC/DC systems with VSCHVDC, the power flow equations are given as follows [14].

Pure AC bus equation

$$
\begin{gathered}
\Delta P_{\mathrm{a} i}=P_{\mathrm{a} i}-U_{\mathrm{a} i} \sum_{j \in i} U_{j}\left(G_{i j} \cos \theta_{i j}+B_{i j} \sin \theta_{i j}\right), \\
\Delta Q_{\mathrm{a} i}=Q_{\mathrm{a} i}-U_{\mathrm{a} i} \sum_{j \in i} U_{j}\left(G_{i j} \sin \theta_{i j}-B_{i j} \cos \theta_{i j}\right) .
\end{gathered}
$$

DC bus equation

$$
\begin{aligned}
\Delta P_{\mathrm{t} i} & =P_{\mathrm{t} i}-U_{\mathrm{t} i} \sum_{j \in i} U_{j}\left(G_{i j} \cos \theta_{i j}+B_{i j} \sin \theta_{i j}\right), \\
\Delta Q_{\mathrm{t} i} & =Q_{\mathrm{t} i}-U_{\mathrm{t} i} \sum_{j \in i} U_{j}\left(G_{i j} \sin \theta_{i j}-B_{i j} \cos \theta_{i j}\right) .
\end{aligned}
$$

VSC converter equation

$$
\begin{aligned}
& \Delta d_{k 1}= P_{\mathrm{t} k}+\frac{\sqrt{6}}{4} M_{k} U_{\mathrm{t} k} U_{\mathrm{d} k}|Y| \cos \left(\delta_{k}+\alpha_{k}\right) \\
&-U_{\mathrm{t} k}^{2}|Y| \cos \alpha_{k}, \\
& \Delta d_{k 2}= Q_{\mathrm{t} k}+\frac{\sqrt{6}}{4} M_{k} U_{\mathrm{t} k} U_{\mathrm{d} k}|Y| \sin \left(\delta_{k}+\alpha_{k}\right) \\
&-U_{\mathrm{t} k}^{2}|Y| \sin \alpha_{k}-\frac{U_{\mathrm{t} k}^{2}}{X_{\mathrm{f} k}} \\
& \Delta d_{k 3}= U_{\mathrm{t} k} I_{\mathrm{d} k}-\frac{\sqrt{6}}{4} M_{k} U_{\mathrm{t} k} U_{\mathrm{d} k}|Y| \cos \left(\delta_{k}-\alpha_{k}\right) \\
&+ \frac{3}{8}\left(M_{k} U_{\mathrm{d} k}\right)^{2}|Y| \cos \alpha_{k}, \\
& \mathbf{I}_{\mathrm{d}}=\mathbf{G}_{\mathrm{d}} \mathbf{U}_{\mathrm{d}} .
\end{aligned}
$$

DC network equation

$$
\Delta d_{k 4}= \pm I_{\mathrm{d} k}-\sum_{s=1}^{n_{\mathrm{AC}}} g_{\mathrm{d} k s} U_{\mathrm{d} s}=0 .
$$

The variables in the equations of (10)-(13) are referenced to the literature [14].

3.2. The Mathematical Description of Newton Method. The mathematical description of multivariable iterative form for Newton method is given by

$$
\begin{gathered}
\Delta x^{(k)}=-\left[F^{\prime}\left(x^{(k)}\right)^{-1}\right] F^{\prime}\left(x^{(k)}\right), \\
x^{(k+1)}=x^{(k)}+\Delta x^{(k)} .
\end{gathered}
$$

The formula (14) has the second-order convergence [23, 28].

The equivalence form of linear equation solution for (14) is given by

$$
\begin{gathered}
{\left[F^{\prime}\left(x^{(k)}\right)\right] \Delta x^{(k)}=-F^{\prime}\left(x^{(k)}\right),} \\
x^{(k+1)}=x^{(k)}+\Delta x^{(k)},
\end{gathered}
$$

where $F^{\prime}\left(x^{(k)}\right)$ is the matrix variable of first-order partial derivative of $F(x)$ and $k$ is the number of iterations.

3.3. The Newton-Type Method of Third-Order Convergence (Algorithm 1). The single variable iterative algorithm format based on modified Newton-type method is given by

$$
x_{k+1}=x_{k}-\frac{f\left(x_{k}+f\left(x_{k}\right) / f^{\prime}\left(x_{k}\right)\right)-f\left(x_{k}\right)}{f^{\prime}\left(x_{k}\right)} .
$$


The iterative format of (16) has the trait of third-order convergence [29]. by

The multivariable matrix equivalent form of (16) is given

$$
\begin{gathered}
F^{\prime}\left(x^{(k)}\right) \Delta x^{(k)}=-F\left(x^{(k)}\right), \\
\tilde{x}^{(k+1)}=x^{(k)}-\Delta x^{(k)}, \\
F^{\prime}\left(x^{(k)}\right) \Delta \tilde{x}^{(k)}=-F^{\prime}\left(\tilde{x}^{(k+1)}\right)+F\left(x^{(k)}\right), \\
x^{(k+1)}=x^{(k)}+\Delta \tilde{x}^{(k)} .
\end{gathered}
$$

The gotten Jacobian matrix and its triangular factorization are being utilized fully in the algorithm iterative process of (17).

3.4. The Newton-Type Method of Third-Order Convergence (Algorithm 2). Another single variable iterative algorithm format with third-order convergence based on Newton-type method is given by:

$$
\begin{aligned}
x_{k+1}= & x_{k}-0.5 f\left(x_{k}\right) \\
& \times\left[\frac{1}{f^{\prime}\left(x_{k}-f\left(x_{k}\right) / f^{\prime}\left(x_{k}\right)\right)}+\frac{1}{f^{\prime}\left(x_{k}\right)}\right] .
\end{aligned}
$$

The iterative format of (18) has the trait of third-order convergence [30]. by:

The multivariable matrix equivalent form of (18) is given

$$
\begin{gathered}
F^{\prime}\left(x^{(k)}\right) \Delta x^{(k)}=-F\left(x^{(k)}\right), \\
\tilde{x}^{(k+1)}=x^{(k)}+\Delta x^{(k)}, \\
F^{\prime}\left(x^{(k+1)}\right) \Delta \tilde{x}^{(k)}=-F\left(x^{(k)}\right), \\
x^{(k+1)}=x^{(k)}+0.5\left(\Delta x^{(k)}+\Delta \tilde{x}^{(k)}\right) .
\end{gathered}
$$

3.5. The Newton-Type Method of Sixth-Order Convergence. For the above presented Algorithm 1 and Algorithm 2, the two iterative formats have the advantages for fast convergence speed of Newton method and less computations of simplified Newton method. The application of Algorithm 1 and Algorithm 2 is a two-step process.

Step 1. The prediction based on the Newton method [23]

$$
\begin{gathered}
\Delta x^{(k)}=-\left[F^{\prime}\left(x^{(k)}\right)\right]^{-1} F\left(x^{(k)}\right), \\
\tilde{x}^{(k+1)}=x^{(k)}+\Delta x^{(k)} .
\end{gathered}
$$

Step 2. The correction for the obtained predicted value of $\tilde{x}^{(k+1)}$

$$
\begin{gathered}
\Delta \tilde{x}^{(k)}=-\left[F^{\prime}\left(x^{(k)}\right)\right]^{-1}\left[F\left(x^{(k)}\right)+F\left(\tilde{x}^{(k+1)}\right)\right], \\
x^{(k+1)}=x^{(k)}+\Delta \tilde{x}^{(k)} .
\end{gathered}
$$

The simplified realization of the iterative procedure for (20) and (21) is given by

$$
\begin{gathered}
y_{k}=x_{k}-\frac{f\left(x_{k}\right)}{f^{\prime}\left(x_{k}\right)}, \\
z_{k}=x_{k}-\frac{2 f\left(x_{k}\right)}{f^{\prime}\left(x_{k}\right)+f^{\prime}\left(y_{k}\right)},
\end{gathered}
$$

where $z_{k}$ is the iterative result of $x_{k+1}$ at the $k$ iterative cycle.

An effective implement of the iterative process of (22) is given by [31]

$$
x_{k+1}=z_{k}-\frac{f\left(z_{k}\right)}{f^{\prime}\left(z_{k}\right)} \text {. }
$$

The approximate value of $f^{\prime}(x)$ at $z_{k}$ is given by

$$
f^{\prime}\left(z_{k}\right) \approx \frac{f^{\prime}\left(x_{k}\right)\left(3 f^{\prime}\left(y_{k}\right)-f^{\prime}\left(x_{k}\right)\right)}{f^{\prime}\left(x_{k}\right)+f^{\prime}\left(y_{k}\right)} .
$$

The (16) or (18), (23), and (24) comprise the new sixthorder convergence method [31]:

$$
\begin{gathered}
y_{k}=x_{k}-\frac{f\left(x_{k}\right)}{f^{\prime}\left(x_{k}\right)}, \\
z_{k}=x_{k}-\frac{2 f\left(x_{k}\right)}{f^{\prime}\left(x_{k}\right)+f^{\prime}\left(y_{k}\right)}, \\
x_{k+1}=z_{k}-\frac{f\left(z_{k}\right)}{f^{\prime}\left(x_{k}\right)} \frac{f^{\prime}\left(x_{k}\right)+f^{\prime}\left(y_{k}\right)}{3 f^{\prime}\left(y_{k}\right)-f^{\prime}\left(x_{k}\right)} .
\end{gathered}
$$

3.6. The Automatic Differentiation Algorithm Based on ThirdOrder Newton-Type Method. The AD technique could always be decomposed to complex computations of basic functions and basic mathematical operations, such as the four arithmetic operations of add, subtract, multiply, and divide, the basic functions of trigonometric function, exponential function, and logarithmic function. Here an instance is given to illustrate the application of $\mathrm{AD}$. The function expression of a certain model is given by

$$
f(x)=\sin x_{1}+e^{x_{1} x_{2}} .
$$

The independent variables and intermediate variables of (26) are given in Table 1. For the use of independent variables and intermediate variables, the function of (26) is decomposed to a series of basic functions. If the value of independent variables is given, the exact value of $y$ is gotten by top-down solution order in Table 1 . Given the value of $\dot{x}_{1}$ and $\dot{x}_{2}$, the differentiation of (26) can be obtained mechanically through the chain rule of differentiation calculation. At present, there are two main modes for the application of $\mathrm{AD}$, the forward mode and the backward mode, as shown in Table 2. And in Table 2, $x_{i, j}=\partial x_{i} / \partial x_{j}, p_{i}=\partial y / \partial x_{i}$.

Now there are two kinds of implementation method for $\mathrm{AD}$, the source code transform method and operator 
TABLE 1: Independent variables and intermediate variables of (26).

\begin{tabular}{lc}
\hline Independent variables & Intermediate variables \\
\hline$x_{1}$ & $x_{3}=\sin x_{1}$ \\
$x_{2}$ & $x_{4}=x_{1} x_{2}$ \\
& $x_{5}=e^{x_{4}}$ \\
& $x_{6}=x_{3}+x_{5}$ \\
& $y=x_{6}$ \\
\hline
\end{tabular}

TABLE 2: Forward and backward mode.

\begin{tabular}{lc}
\hline Forward mode & Backward mode \\
\hline$\dot{x}_{3}=\operatorname{con}\left(x_{1}\right) \cdot \dot{x}_{1}$ & $p_{1}=p_{1}+x_{3,1} p_{3}$ \\
$\dot{x_{4}}=\dot{x}_{1} x_{2}+x_{1} \dot{x_{2}}$ & $p_{1}=p_{1}+x_{4,1} p_{4}, p_{2}=p_{2}+x_{4,2} p_{4}$ \\
$\dot{x_{5}}=x_{5} \dot{x}_{4}$ & $p_{4}=p_{4}+x_{5,4} p_{5}$ \\
$\dot{x_{6}}=\dot{x}_{3}+\dot{x}_{5}$ & $p_{3}=p_{3}+x_{6,3} p_{6}, p_{5}=p_{5}+x_{6,5} p_{6}$ \\
$\dot{y}=\dot{x}_{6}$ & $p_{7}=1$ \\
\hline
\end{tabular}

overloading method. The typical representative softwares for the former is ADIFOR and ADIC. The typical representative software for the latter is ADOL-C and ADC. The method of ADOL-C realizes the differentiation of $\mathrm{C}++$ program automatically by using operator overloading and can calculate any order derivative by forward and backward mode. In this paper, the ADOL-C method is used to realize the differential operation [32].

The steps of the improved AD algorithm based on thirdorder Newton method are listed below.

Step 1. Read network parameter, including bus number, active and reactive power of load, compensate capacitance, branch number of line, resistor and reactance in series, and ratio and impedance of transformer.

Step 2 (initialization). Form the admittance matrix of the DC and AC systems.

Step 3. Distribute space for AD and state active variables, including independent variables and dependent variables.

Step 4. Transmit the value of system variable to active variable.

Step 5. Form the expression of dependent variable by using independent variable.

Step 6. Judge the maximum of imbalance equation whether to meet the error precision or not. If yes, exit the loop. If not, the loop goes on.

Step 7. Call the function of Jacobian and Hessian of AD.

Step 8. Solve the equation of (17) or (19).

Return to Step 3.

\section{Case Studies}

In this part, in order to validate the correctness and suitability of the proposed algorithms, three sections are presented.

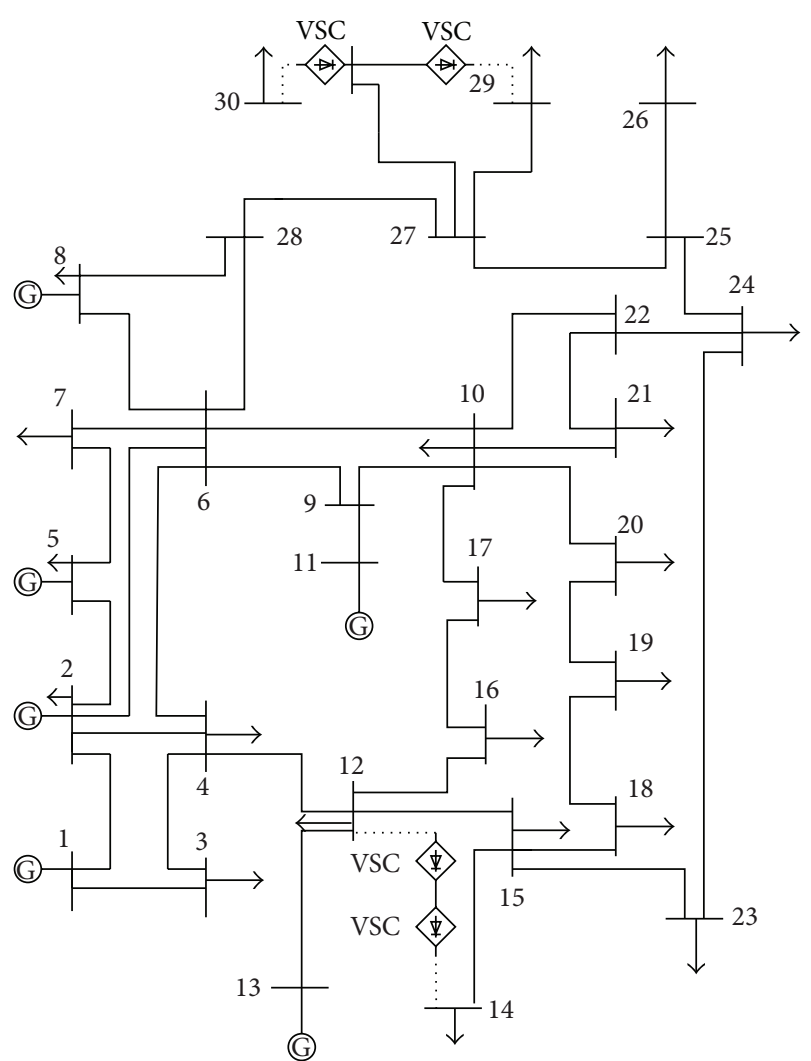

FIgUre 2: The modified IEEE-30 bus AC/DC system with VSCHVDC.

(1) For the modified high-order Newton methods, the modified IEEE 30-bus system with two-terminal and multi-infeed VSC-HVDC is analyzed in detail firstly.

(2) Then the simulation results of performance comparisons for the improved high-order Newton methods are presented among the modified IEEE 5-bus, IEEE 9-bus, IEEE 14-bus, IEEE 57-bus, and IEEE 118- bus text systems.

(3) At last, the AD based on third-order Newton method is evaluated for the modified IEEE 30-bus system with two-terminal of VSC-HVDC.

4.1. The Modified IEEE 30-Bus System with Two-Terminal and Multi-Infeed VSC-HVDC. The proposed method has been applied to the modified IEEE 30-bus system [33]. The wiring diagram is shown in Figure 2. In this section, two cases are considered and compared. In Figure 2, the dotted lines represent the possible positions of VSC stations, and the specific positions are located as follows.

(1) In the system with two-terminal VSC-HVDC, the VSC1 and VSC2 are connected to AC line of bus 29 and bus 30 , respectively.

(2) In the system with two-infeed VSC-HVDC, the VSC1, VSC2, VSC3, and VSC4 are connected to AC line of bus 12 , bus 14 , bus 29 , and bus 30 , respectively. 
TABLE 3: Results of the power flow calculation of AC system.

\begin{tabular}{|c|c|c|c|c|c|c|c|c|c|}
\hline \multirow{3}{*}{ Control mode } & \multirow{3}{*}{ Method } & \multicolumn{8}{|c|}{ Bus Number } \\
\hline & & \multicolumn{2}{|c|}{1} & \multicolumn{2}{|c|}{2} & \multicolumn{2}{|c|}{3} & \multicolumn{2}{|c|}{4} \\
\hline & & $V$ & $\theta$ & $V$ & $\theta$ & $V$ & $\theta$ & $V$ & $\theta$ \\
\hline \multirow{4}{*}{ (1) $+(3)$} & Newton & 1.0600 & 0 & 1.0450 & -5.5025 & 1.0333 & -8.1365 & 1.0271 & -9.8132 \\
\hline & Algorithm 1 & 1.0600 & 0 & 1.0450 & -5.5025 & 1.0333 & -8.1365 & 1.0271 & -9.8132 \\
\hline & Algorithm 2 & 1.0600 & 0 & 1.0450 & -5.5025 & 1.0333 & -8.1365 & 1.0271 & -9.8132 \\
\hline & Sixth-order Newton & 1.0600 & 0 & 1.0450 & -5.5025 & 1.0333 & -8.1365 & 1.0271 & -9.8132 \\
\hline \multirow{4}{*}{ (1) + (4) } & Newton & 1.0600 & 0 & 1.0450 & -5.5012 & 1.0340 & -8.1415 & 1.0279 & -9.8187 \\
\hline & Algorithm 1 & 1.0600 & 0 & 1.0450 & -5.5012 & 1.0340 & -8.1415 & 1.0279 & -9.8187 \\
\hline & Algorithm 2 & 1.0600 & 0 & 1.0450 & -5.5012 & 1.0340 & -8.1415 & 1.0279 & -9.8187 \\
\hline & Sixth-order Newton & 1.0600 & 0 & 1.0450 & -5.5012 & 1.0340 & -8.1415 & 1.0279 & -9.8187 \\
\hline \multirow{4}{*}{ (2) $+(3)$} & Newton & 1.0600 & 0 & 1.0450 & -5.5013 & 1.0341 & -8.1434 & 1.0281 & -9.8208 \\
\hline & Algorithm 1 & 1.0600 & 0 & 1.0450 & -5.5013 & 1.0341 & -8.1434 & 1.0281 & -9.8208 \\
\hline & Algorithm 2 & 1.0600 & 0 & 1.0450 & -5.5013 & 1.0341 & -8.1434 & 1.0281 & -9.8208 \\
\hline & Sixth-order Newton & 1.0600 & 0 & 1.0450 & -5.5013 & 1.0341 & -8.1434 & 1.0281 & -9.8208 \\
\hline \multirow{4}{*}{ (2) + (4) } & Newton & 1.0600 & 0 & 1.0450 & -5.4996 & 1.0344 & -8.1435 & 1.0284 & -9.8208 \\
\hline & Algorithm 1 & 1.0600 & 0 & 1.0450 & -5.4996 & 1.0344 & -8.1435 & 1.0284 & -9.8208 \\
\hline & Algorithm 2 & 1.0600 & 0 & 1.0450 & -5.4996 & 1.0344 & -8.1435 & 1.0284 & -9.8208 \\
\hline & Sixth-order Newton & 1.0600 & 0 & 1.0450 & -5.4996 & 1.0344 & -8.1435 & 1.0284 & -9.8208 \\
\hline
\end{tabular}

TABLE 4: Results of the power flow calculation of DC system.

\begin{tabular}{|c|c|c|c|c|c|c|}
\hline \multirow{2}{*}{\multicolumn{2}{|c|}{ DC variable }} & \multirow{2}{*}{ Converter number } & \multicolumn{4}{|c|}{ Control mode } \\
\hline & & & (1) + (3) & (1) + (4) & (2) + (3) & (2) + (4) \\
\hline \multirow{2}{*}{\multicolumn{2}{|c|}{ Newton }} & $\mathrm{VSC}_{1}$ & 2.0000 & 2.0000 & 2.0000 & 2.0000 \\
\hline & & $\mathrm{VSC}_{2}$ & 1.9994 & 1.9994 & 1.9994 & 1.9994 \\
\hline \multirow{6}{*}{$U_{d}$} & \multirow{2}{*}{ Algorithm 1} & $\mathrm{VSC}_{1}$ & 2.0000 & 2.0000 & 2.0000 & 2.0000 \\
\hline & & $\mathrm{VSC}_{2}$ & 1.9994 & 1.9994 & 1.9994 & 1.9994 \\
\hline & \multirow{2}{*}{ Algorithm 2} & $\mathrm{VSC}_{1}$ & 2.0000 & 2.0000 & 2.0000 & 2.0000 \\
\hline & & $\mathrm{VSC}_{2}$ & 1.9994 & 1.9994 & 1.9994 & 1.9994 \\
\hline & \multirow{2}{*}{ Sixth-order Newton } & $\mathrm{VSC}_{1}$ & 2.0000 & 2.0000 & 2.0000 & 2.0000 \\
\hline & & $\mathrm{VSC}_{2}$ & 1.9994 & 1.9994 & 1.9994 & 1.9994 \\
\hline \multirow{8}{*}{$\delta$} & \multirow{2}{*}{ Newton } & $\mathrm{VSC}_{1}$ & 0.3556 & 0.3402 & 0.3381 & 0.3322 \\
\hline & & $\mathrm{VSC}_{2}$ & -0.3701 & -0.2960 & -0.3495 & -0.3047 \\
\hline & \multirow{2}{*}{ Algorithm 1} & $\mathrm{VSC}_{1}$ & 0.0062 & 0.0059 & 0.0059 & 0.0058 \\
\hline & & $\mathrm{VSC}_{2}$ & -0.0065 & -0.0052 & -0.0061 & -0.0053 \\
\hline & \multirow{2}{*}{ Algorithm 2} & $\mathrm{VSC}_{1}$ & 0.0062 & 0.0059 & 0.0059 & 0.0058 \\
\hline & & $\mathrm{VSC}_{2}$ & -0.0065 & -0.0052 & -0.0061 & -0.0053 \\
\hline & \multirow{2}{*}{ Sixth-order Newton } & $\mathrm{VSC}_{1}$ & 0.0062 & 0.0059 & 0.0059 & 0.0058 \\
\hline & & $\mathrm{VSC}_{2}$ & -0.0065 & -0.0052 & -0.0061 & -0.0053 \\
\hline \multirow{8}{*}{$M$} & \multirow{2}{*}{ Newton } & $\mathrm{VSC}_{1}$ & 0.7663 & 0.7838 & 0.8282 & 0.8257 \\
\hline & & $\mathrm{VSC}_{2}$ & 0.7574 & 0.8188 & 0.7794 & 0.8161 \\
\hline & \multirow{2}{*}{ Algorithm 1} & $\mathrm{VSC}_{1}$ & 0.7663 & 0.7838 & 0.8282 & 0.8257 \\
\hline & & $\mathrm{VSC}_{2}$ & 0.7574 & 0.8188 & 0.7794 & 0.8161 \\
\hline & \multirow{2}{*}{ Algorithm 2} & $\mathrm{VSC}_{1}$ & 0.7663 & 0.7838 & 0.8282 & 0.8257 \\
\hline & & $\mathrm{VSC}_{2}$ & 0.7574 & 0.8188 & 0.7794 & 0.8161 \\
\hline & \multirow{2}{*}{ Sixth-order Newton } & $\mathrm{VSC}_{1}$ & 0.7663 & 0.7838 & 0.8282 & 0.8257 \\
\hline & & $\mathrm{VSC}_{2}$ & 0.7574 & 0.8188 & 0.7794 & 0.8161 \\
\hline
\end{tabular}


TABLE 5: Comparisons of iteration times and computing time.

\begin{tabular}{lcccccccc}
\hline \multirow{2}{*}{ Control mode } & \multicolumn{4}{c}{ Iteration times } & \multicolumn{4}{c}{ Computing time (ms) } \\
& Newton & Algorithm 1 & Algorithm 2 & Sixth-order Newton & Newton & Algorithm 1 & Algorithm 2 & Sixth-order Newton \\
\hline (1) + (3) & 4 & 3 & 3 & 2 & 8.6885 & 8.8367 & 0.1040 & 12.6763 \\
(1) + (4) & 4 & 3 & 3 & 2 & 9.8464 & 5.6506 & 0.1023 & 9.5408 \\
(2) + (3) & 4 & 3 & 3 & $1.5^{*}$ & 9.3356 & 5.6121 & 0.1045 & 8.4520 \\
(2) + (4) & 4 & 3 & 3 & $1.5^{*}$ & 9.4767 & 5.6403 & 0.0990 & 8.2271 \\
\hline
\end{tabular}

TABLE 6: Comparison of iteration times and computing time.

\begin{tabular}{|c|c|c|c|c|c|c|c|c|c|}
\hline Contr & ol mode & & Iter & ation times & & & Compu & ting time (ms) & \\
\hline $\mathrm{VSC}_{1}+\mathrm{VSC}_{2}$ & $\mathrm{VSC}_{3}+\mathrm{VSC}_{4}$ & Newton & Algorithm 1 & Algorithm 2 & $\begin{array}{l}\text { Sixth-order } \\
\text { Newton }\end{array}$ & Newton & Algorithm 1 & Algorithm 2 & $\begin{array}{c}\text { Sixth-order } \\
\text { Newton }\end{array}$ \\
\hline (1) + (3) & (1) + (3) & 4 & 3 & 3 & 1.5 & 10.0649 & 8.6786 & 0.1186 & 0.4648 \\
\hline (1) + (3) & (1) + (4) & 4 & 3 & 3 & 1.5 & 9.4347 & 6.8694 & 0.1110 & 0.4870 \\
\hline (1) + (3) & (2) + (3) & 4 & 3 & 3 & 1.5 & 9.4438 & 6.0041 & 0.1086 & 0.4399 \\
\hline (1) + (3) & (2) + (4) & 4 & 3 & 3 & 1.5 & 9.8407 & 6.7326 & 0.1136 & 0.4384 \\
\hline (1) + (4) & (1) + (4) & 4 & 3 & 3 & 1.5 & 9.4073 & 6.3733 & 0.1164 & 0.4885 \\
\hline (1) + (4) & (2) + (3) & 4 & 3 & 3 & 1.5 & 10.1531 & 5.9505 & 0.1113 & 0.4499 \\
\hline (1) + (4) & (2) + (4) & 4 & 3 & 3 & 1.5 & 5.8884 & 6.0551 & 0.1121 & 0.4273 \\
\hline (2) + (3) & (2) $+(3)$ & 4 & 3 & 3 & 1.5 & 9.7429 & 5.8435 & 0.1330 & 0.4283 \\
\hline (2) + (3) & (2) + (4) & 4 & 3 & 3 & 1.5 & 9.8400 & 6.1026 & 0.1216 & 0.3860 \\
\hline (2) + (4) & (2) + (4) & 4 & 3 & 3 & 1.5 & 9.7773 & 6.2020 & 0.1168 & 0.4181 \\
\hline
\end{tabular}

4.1.1. The Modified IEEE 30-Bus System with Two-Terminal VSC-HVDC. The results of the power flow calculation of the AC system and DC system under different control modes for Newton, third-order and sixth-order Newton methods are shown in Tables 3 and 4 . In Table 3, the simulation results of bus number of 1,2,3, and 4 are presented only, and the other buses of the modified IEEE 30-bus system are not included for the sake of brevity. There are two methods of third-order Newton in Tables 3 and 4, Algorithm 1, and Algorithm 2. In Table 3, the "Newton" method is the algorithm in the references of [5-10], the unit of $V$ is p.u., and the unit of $\theta$ is ${ }^{\circ}$.

It can be seen from Table 3 the voltage amplitudes of bus 1 and bus 2 are all the same for the proposed four methods. This is due to the node type of bus 1 and bus 2 for the IEEE 30 -bus system. Bus 1 is the equilibrium node. And Bus 2 is the $\mathrm{PV}$ node. So in the iterative process of the proposed different methods, if the generators of bus 1 and bus 2 have not reached the limit of reactive power, the voltage amplitudes of bus 1 and bus 2 remain the same.

In Table 4, the DC variables of Ud are the same for different control modes and Newton methods; the reason is that for the general used four control modes of VSC, all the mode combinations of (1) + (3), (1) + (4), (2) + (3), and (2) + (4) contain the constant DC voltage control category. As a result, in the operation process of VSC-HVDC system, the DC voltage of VSC remains constant.

Both in Tables 3 and 4, for the proposed algorithms of Newton, Algorithm 1, Algorithm 2, and sixth-order Newton method, the results of the power flow calculation for the four different control modes remain the same fundamentally. And the operational parameters of DC system are all in the normal range. The simulation results also illustrate the flexible application of the third-order and sixth-order Newton methods for the AC/DC system with VSC-HVDC.

The comparisons of iteration times and computing time for the four proposed Newton methods are shown in Table 5. In Table 5, the ${ }^{*}$ indicates that the criterion for convergence is met only at the front half iteration procedure. As seen in Table 5, for the modified IEEE 30-bus text system with twoterminal VSC-HVDC, the iteration times of sixth-order Newton method are evidently less than other Newton methods. And the CPU computing time of the third-order Newton of Algorithm 2 is smaller than other Newton methods under four different control modes. The reason is that for the modified IEEE 30-bus text system, the computation task of Jacobian matrix formation and triangular factorization for high-order Newton method is less than Newton method.

4.1.2. The Modified IEEE 30-Bus System with Multi-Infeed VSC-HVDC. For the flexible control performance and particular technical advantages, the VSC-HVDC is suitable for application in multi-infeed system $[5,34]$. In this section, the comparison of iteration times and computing time under different control modes for improved Newton methods is shown in Table 6 for the modified IEEE 30-bus system with two-infeed VSC-HVDC. For two-infeed VSC-HVDC, the combinations ways of VSC have ten different types, as shown in Table 6.

It can be seen from Table 6, both for the iteration times and the computing time, the high-order Newton-type of 
TABLE 7: The topology and parameter settings for different IEEE text systems.

\begin{tabular}{|c|c|c|c|c|c|c|c|c|c|c|c|c|c|c|c|c|c|c|c|c|}
\hline \multirow{2}{*}{$\begin{array}{l}\text { Topology } \\
\text { Modified } \\
\text { test system }\end{array}$} & \multicolumn{10}{|c|}{ Two-terminal } & \multirow{2}{*}{\multicolumn{4}{|c|}{$\begin{array}{l}\text { Two-infeed } \\
\text { IEEE-14 }\end{array}$}} & \multicolumn{6}{|c|}{ Three-terminal } \\
\hline & & E-5 & & E-9 & IEE] & E-14 & IEEI & E-57 & IEEF & & & & & & & IEEE-14 & & & IEEE-57 & \\
\hline \multirow{2}{*}{ Bus number } & $\mathrm{VSC}_{1}$ & $\mathrm{VSC}_{2}$ & $\mathrm{VSC}_{1}$ & $\mathrm{VSC}_{2}$ & $\mathrm{VSC}_{1}$ & $\mathrm{VSC}_{2}$ & $\mathrm{VSC}_{1}$ & $\mathrm{VSC}_{2}$ & $\mathrm{VSC}_{1}$ & $\mathrm{VSC}_{2}$ & $\mathrm{VSC}_{1}$ & $\mathrm{VSC}_{2}$ & $\mathrm{VSC}_{3}$ & $\mathrm{VSC}_{4}$ & $\mathrm{VSC}_{1}$ & $\mathrm{VSC}_{2}$ & $\mathrm{VSC}_{3}$ & $\mathrm{VSC}_{1}$ & $\mathrm{VSC}_{2}$ & $\mathrm{VSC}_{3}$ \\
\hline & 4 & 5 & 8 & 9 & 13 & 14 & 56 & 57 & 75 & 118 & 12 & 14 & 29 & 30 & 12 & 13 & 14 & 55 & 56 & 41 \\
\hline
\end{tabular}

TABLE 8: Performance comparison for different text systems.

\begin{tabular}{lccccccc}
\hline \multirow{2}{*}{ Topology } & \multirow{2}{*}{ Modified test system } & \multicolumn{3}{c}{ Iteration times } & \multicolumn{3}{c}{ Computing time (ms) } \\
& & Newton & Algorithm 1 & Sixth-order Newton & Newton & Algorithm 1 & Sixth-order Newton \\
\hline Two-terminal & IEEE-5 & 5 & 4 & 1.5 & 4.8287 & 4.4651 & 5.3692 \\
Two-terminal & IEEE-9 & 4 & 3 & 1.5 & 3.1646 & 2.2094 & 2.7866 \\
Two-terminal & IEEE-14 & 4 & 3 & 1.5 & 4.1375 & 2.6509 & 4.1890 \\
Two-infeed & IEEE-14 & 4 & 3 & 1.5 & 4.3529 & 3.1544 & 3.9907 \\
Three-terminal & IEEE-14 & 4 & 3 & 1.5 & 4.3546 & 2.9592 & 4.1260 \\
Two-terminal & IEEE-57 & 4 & 4 & 2 & 22.0698 & 14.2854 & 23.6236 \\
Three-terminal & IEEE-57 & 4 & 4 & 2 & 28.9858 & 24.7143 & 29.0658 \\
Two-terminal & IEEE-118 & 4 & 3 & 1.5 & 120.8938 & 70.8048 & 115.1420 \\
\hline
\end{tabular}

TABLE 9: Results of the power flow calculation of DC system.

\begin{tabular}{|c|c|c|c|}
\hline & DC variable & $\begin{array}{c}\text { Converter } \\
\text { number }\end{array}$ & $\begin{array}{c}\text { Control modes } \\
\text { of (1) + (3) }\end{array}$ \\
\hline \multirow{4}{*}{$U_{d}$} & \multirow{2}{*}{ Algorithm 1 + AD } & $\mathrm{VSC}_{1}$ & 2.0000 \\
\hline & & $\mathrm{VSC}_{2}$ & 1.9994 \\
\hline & \multirow{2}{*}{ Algorithm $2+\mathrm{AD}$} & $\mathrm{VSC}_{1}$ & 2.0000 \\
\hline & & $\mathrm{VSC}_{2}$ & 1.9994 \\
\hline \multirow{4}{*}{$\delta$} & \multirow{2}{*}{ Algorithm 1 + AD } & $\mathrm{VSC}_{1}$ & 0.0062 \\
\hline & & $\mathrm{VSC}_{2}$ & -0.0064 \\
\hline & \multirow{2}{*}{ Algorithm $2+\mathrm{AD}$} & $\mathrm{VSC}_{1}$ & 0.0062 \\
\hline & & $\mathrm{VSC}_{2}$ & -0.0064 \\
\hline \multirow{4}{*}{$M$} & \multirow{2}{*}{ Algorithm 1 + AD } & $\mathrm{VSC}_{1}$ & 0.7677 \\
\hline & & $\mathrm{VSC}_{2}$ & 0.7589 \\
\hline & \multirow[t]{2}{*}{ Algorithm $2+\mathrm{AD}$} & $\mathrm{VSC}_{1}$ & 0.7677 \\
\hline & & $\mathrm{VSC}_{2}$ & 0.7589 \\
\hline
\end{tabular}

third-order and sixth-order Newton methods is less than the Newton method. And the advantage of computing time for Algorithm 2 is obvious. Table 6 also shows the proposed highorder methods suitable for the AC/DC systems with multiinfeed VSC-HVDC.

4.2. The Simulations of Modified IEEE 5-, 9-, 14-, 57-, and 118-Bus Systems. The modified IEEE 5-, 9-, 14-, 57-, and 118bus systems are analyzed in this section [33]. The topology and parameter settings for those different IEEE text systems are shown in Table 7 . The simulation results of performance comparisons for those IEEE text systems among improved Newton methods are shown in Table 8 . The system topologies of two-terminal, two-infeed, and three-terminal are analyzed in this section.
It can be seen in Table 8, as the size and scale of the IEEE text systems grow, the iteration times keep mostly unchangeable for the third-order Newton and sixth-order Newton methods. And the computing time of the third-order or sixth-order Newton method is less than the Newton method. The validity and usability of the proposed improved Newton method suitable for VSC-HVDC system are certified.

\subsection{The Simulation Results of AD Based on Third-Order} Newton Method. In this part, the simulation results for AD algorithm based on Algorithm 1 and Algorithm 2 of third-order Newton method are presented. The IEEE-30 bus text system with two-terminal VSC-HVDC is employed to demonstrate the validity of the proposed $\mathrm{AD}$ algorithm. The results of the power flow calculation of DC system of control mode (1) + (3) are shown in Table 9. The comparison of iteration times and computing time for the proposed $\mathrm{AD}$ algorithm is shown in Table 10.

From the results of Tables 9 and 10, the following can be seen.

(1) Compared with the results of Algorithm 1 and Algorithm 2 of third-order Newton method, as shown in Table 4, the improved AD algorithm satisfies the operation requirements of VSC parameter.

(2) Compared with the results of Table 5, for the iteration times and computing time, the improved $\mathrm{AD}$ algorithm has certain advantages.

(3) The result shows that $\mathrm{AD}$ technology is suitable for use in the third-order Newton method of VSCHVDC system. And the application of AD technology reduces the work of hand code greatly. The efficiency of code programming is improved. 
TABLE 10: Comparison of iteration times and computing time

\begin{tabular}{|c|c|c|c|c|}
\hline \multirow{2}{*}{$\begin{array}{l}\text { Control mode } \\
\% \mathrm{VSC}_{1}+\mathrm{VSC}_{2}\end{array}$} & \multicolumn{2}{|c|}{ Iteration times } & \multicolumn{2}{|c|}{ Computing time (ms) } \\
\hline & Algorithm $1+\mathrm{AD}$ & Algorithm $2+\mathrm{AD}$ & Algorithm $1+\mathrm{AD}$ & Algorithm $2+\mathrm{AD}$ \\
\hline (1) + (3) & 2 & 2 & 0.15 & 0.31 \\
\hline (1) + (4) & 2 & 2 & 0.31 & 0.31 \\
\hline (2) + (3) & 2 & 2 & 0.31 & 0.32 \\
\hline (2) + (4) & 2 & 2 & 0.31 & 0.32 \\
\hline
\end{tabular}

\section{Conclusions}

In this paper, based on the steady mathematical model of VSC-HVDC, the modified third-order Newton and sixthorder Newton methods have been presented to calculate the power flow of AC/DC systems with VSC-HVDC. The multivariate iteration matrix forms of the presented algorithms suitable for VSC-HVDC system are given. The proposed high-order Newton method has the third-order and sixthorder convergence, without solving the Hessian matrix. The task of the calculation is greatly reduced, and the efficiency is improved. Based on the third-order Newton method, the automatic differentiation technology is used to increase the efficiency of hand code. Some numerical examples on the modified IEEE bus systems with two-terminal, multiterminal, and multi-infeed VSC-HVDC have demonstrated the computational performance of the power flow algorithms with incorporation of VSC-HVDC models.

\section{Acknowledgments}

This work is supported in part by the National Natural Science Foundation of China (Grant no. 61104045 and U1204506), in part by the Youth Project of National Social Science Fund (Grant no. 09CJY007), and in part by the Fundamental Research Funds for the Central Universities of China (Grant no. 2012B03514).

\section{References}

[1] N. Flourentzou, V. G. Agelidis, and G. D. Demetriades, "VSCbased HVDC power transmission systems: an overview," IEEE Transactions on Power Electronics, vol. 24, no. 3, pp. 592-602, 2009.

[2] P. Haugland, It's Time to Connect-Technical Description of HVDC Light Technology, ABB, 2008.

[3] A. L. P. de Oliveira, C. E. Tibúrcio, M. N. Lemes, and D. Retzmann, "Prospects of Voltage-Sourced Converters (VSC) applications in DC transmission systems," in Proceedings of the IEEE/PES Transmission and Distribution Conference and Exposition: Latin America (T and D-LA '10), pp. 491-495, Sao Paulo, Brazil, November 2010.

[4] J. M. Maza-Ortega, A. Gomez-Exposito, M. Barragan-Villarejo, E. Romero-Ramos, and A. Marano-Marcolini, "Voltage source converter-based topologies to further integrate renewable energy sources in distribution systems," IET Renewable Power Generation, vol. 6, no. 6, pp. 435-445, 2012.

[5] X. P. Zhang, "Multiterminal voltage-sourced converter-based HVDC models for power flow analysis," IEEE Transactions on Power Systems, vol. 19, no. 4, pp. 1877-1884, 2004.
[6] C. Angeles-Camacho, O. L. Tortelli, E. Acha, and C. R. FuerteEsquivel, "Inclusion of a high voltage DC-voltage source converter model in a Newton-Raphson power flow algorithm," IEE Proceedings: Generation, Transmission and Distribution, vol. 150, no. 6, pp. 691-696, 2003.

[7] C. Zheng, X. Zhou, R. Li, and C. Sheng, "Study on the steady characteristic and algorithm of power flow for VSC-HVDC," Proceedings of the CSEE, vol. 25, no. 6, pp. 1-5, 2005.

[8] A. Pizano-Martinez, C. R. Fuerte-Esquivel, H. Ambriz-Pérez, and E. Acha, "Modeling of VSC-based HVDC systems for a Newton-Raphson OPF algorithm," IEEE Transactions on Power Systems, vol. 22, no. 4, pp. 1794-1803, 2007.

[9] A. Pizano-Martinez, C. R. Fuerte-Esquivel, and C. AngelesCamacho, "Voltage source converter based high-voltage DC system modeling for optimal power flow studies," Electric Power Components and Systems, vol. 40, no. 3, pp. 312-320, 2012.

[10] C. Liu, B. Zhang, Y. Hou, F. F. Wu, and Y. Liu, "An improved approach for AC-DC power flow calculation with multi-infeed DC systems," IEEE Transactions on Power Systems, vol. 26, no. 2, pp. 862-869, 2011.

[11] X. Wang, Y. Song, and M. Irving, Modern Power Systems Analysis, Springer, Berlin, Germany, 2008.

[12] J. Beerten, S. Cole, and R. Belmans, "Generalized steady-state vsc mtdc model for sequential ac/dc power flow algorithms," IEEE Transactions on Power Systems, vol. 27, no. 2, pp. 821-829, 2012.

[13] T. Smed, G. Andersson, G. B. Sheble, and L. L. Grigsby, "A new approach to AC/DC power flow," IEEE Transactions on Power Systems, vol. 6, no. 3, pp. 1238-1244, 1991.

[14] Y. Wei, Z. Zheng, Y. Sun, Z. Wei, and G. Sun, "Voltage stability bifurcation analysis for AC/DC systems with VSC-HVDC," Abstract and Applied Analysis, vol. 2013, Article ID 387167, 9 pages, 2013.

[15] C. R. Fuerte-Esquivel, E. Acha, and H. Ambriz-Pérez, "A comprehensive Newton-Raphson UPFC model for the quadratic power flow solution of practical power networks," IEEE Transactions on Power Systems, vol. 15, no. 1, pp. 102-109, 2000.

[16] H. Ambriz-Perez, E. Acha, and C. R. Fuerte-Esquivel, "Advanced SVC models for Newton-Raphson load flow and Newton optimal power flow studies," IEEE Transactions on Power Systems, vol. 15, no. 1, pp. 129-136, 2000.

[17] C. R. Fuerte-Esquivel and E. Acha, "A Newton-type algorithm for the control of power flow in electrical power networks," IEEE Transactions on Power Systems, vol. 12, no. 4, pp. 1474-1480, 1997.

[18] M. A. Noor, K. I. Noor, E. Al-Said, and M. Waseem, "Some new iterative methods for nonlinear equations," Mathematical Problems in Engineering, vol. 2010, Article ID 198943, 12 pages, 2010.

[19] P. Tang and X. Wang, "An iteration method with generally convergent property for cubic polynomials," International Journal of Bifurcation and Chaos, vol. 19, no. 1, pp. 395-401, 2009. 
[20] A. Cordero, J. L. Hueso, E. Martínez, and J. R. Torregrosa, "Increasing the convergence order of an iterative method for nonlinear systems," Applied Mathematics Letters, vol. 25, no. 12, pp. 2369-2374, 2012.

[21] D. Herceg and D. Herceg, "Means based modifications of Newton's method for solving nonlinear equations," Applied Mathematics and Computation, vol. 219, no. 11, pp. 6126-6133, 2013.

[22] F. Soleymani, S. Karimi Vanani, and A. Afghani, "A general three-step class of optimal iterations for nonlinear equations," Mathematical Problems in Engineering, vol. 2011, Article ID 469512, 10 pages, 2011.

[23] Z. Y. Sun, Y. Sun, and W. H. Ning, "Power flow algorithm with cubic convergence," Power System Protection and Control, vol. 37, no. 4, pp. 5-8+28, 2009.

[24] T. Orfanogianni and R. Bacher, "Using automatic code differentiation in power flow algorithms," IEEE Transactions on Power Systems, vol. 14, no. 1, pp. 138-144, 1999.

[25] M. Bartholomew-Biggs, S. Brown, B. Christianson, and L. Dixon, "Automatic differentiation of algorithms," Journal of Computational and Applied Mathematics, vol. 124, no. 1-2, pp. 171-190, 2000.

[26] Q. Jiang, G. Geng, C. Guo, and Y. Cao, "An efficient implementation of automatic differentiation in interior point optimal power flow," IEEE Transactions on Power Systems, vol. 25, no. 1, pp. 147$155,2010$.

[27] S. Cole, Steady-State and Dynamic Modeling of VSC-HVDC Systems for Power System Simulation, K. U. Leuven, Leuven, Belgium, 2010.

[28] A. M. Ostrowski, Solution of Equations in Euclidean and Banach Spaces, Academic Press, New York, NY, USA, 1973.

[29] J. Kou, Y. Li, and X. Wang, "A modification of Newton method with third-order convergence," Applied Mathematics and Computation, vol. 181, no. 2, pp. 1106-1111, 2006.

[30] H. H. H. Homeier, "On Newton-type methods with cubic convergence," Journal of Computational and Applied Mathematics, vol. 176, no. 2, pp. 425-432, 2005.

[31] S. K. Parhi and D. K. Gupta, "A sixth order method for nonlinear equations," Applied Mathematics and Computation, vol. 203, no. 1, pp. 50-55, 2008.

[32] A. Walther and A. Griewank, A Package for the Automatic Differentiation of Algorithms Written in $\mathrm{C} / \mathrm{C}++, 2009$, http://www.coin-or.org/projects/ADOL-C.xml.

[33] Power Systems Test Case Archive, University of Washington, http://www.ee.washington.edu/research/pstca/.

[34] Y. Wei, Z. Wei, and G. Sun, "A commentary on voltage stability of multi-infeed AC/DC system with VSC-HDVC," in Proceedings of the Asia-Pacific Power and Energy Engineering Conference (APPEEC '11), pp. 1-4, Wuhan, China, March 2011. 


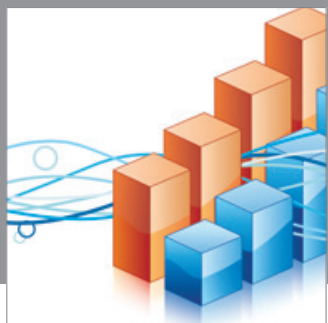

Advances in

Operations Research

mansans

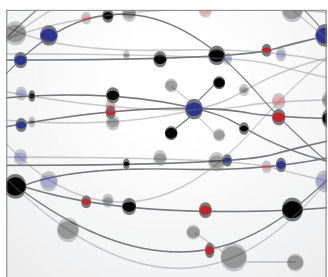

The Scientific World Journal
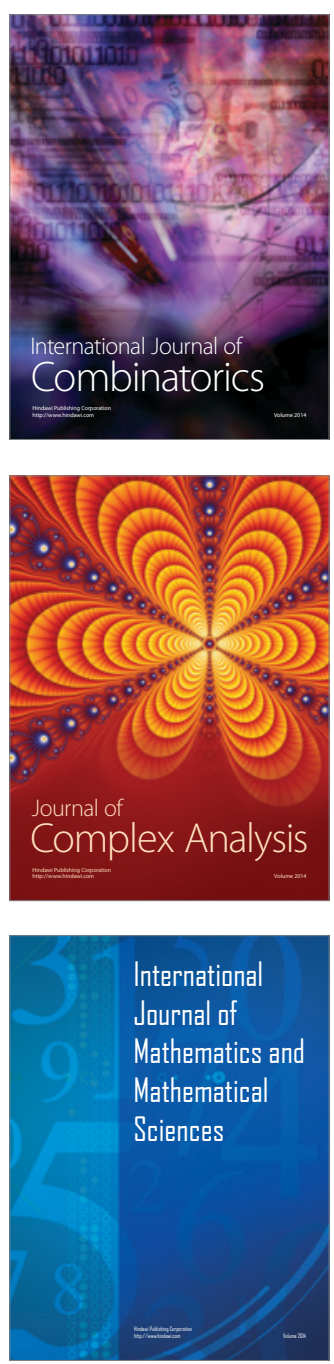
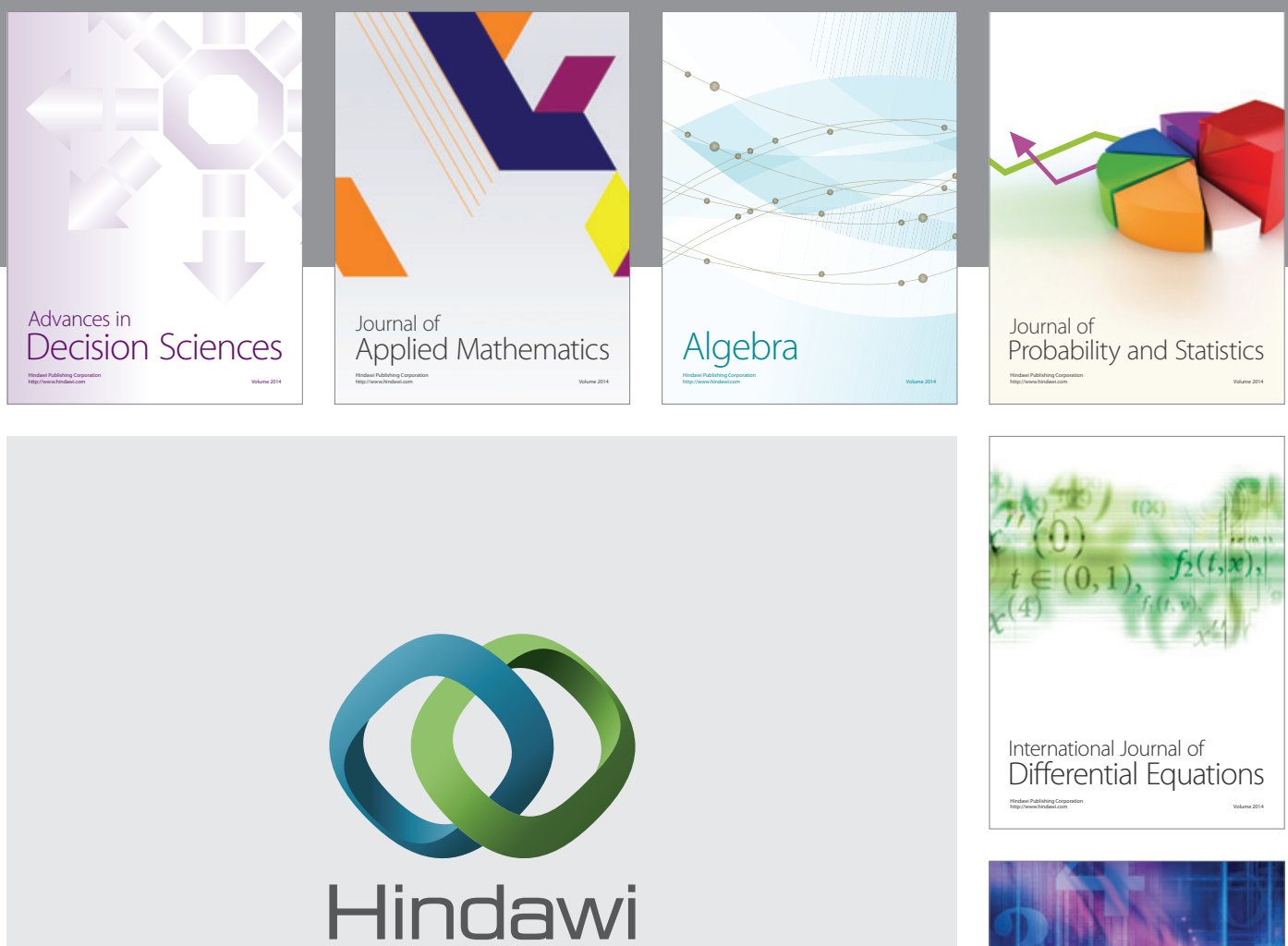

Submit your manuscripts at http://www.hindawi.com
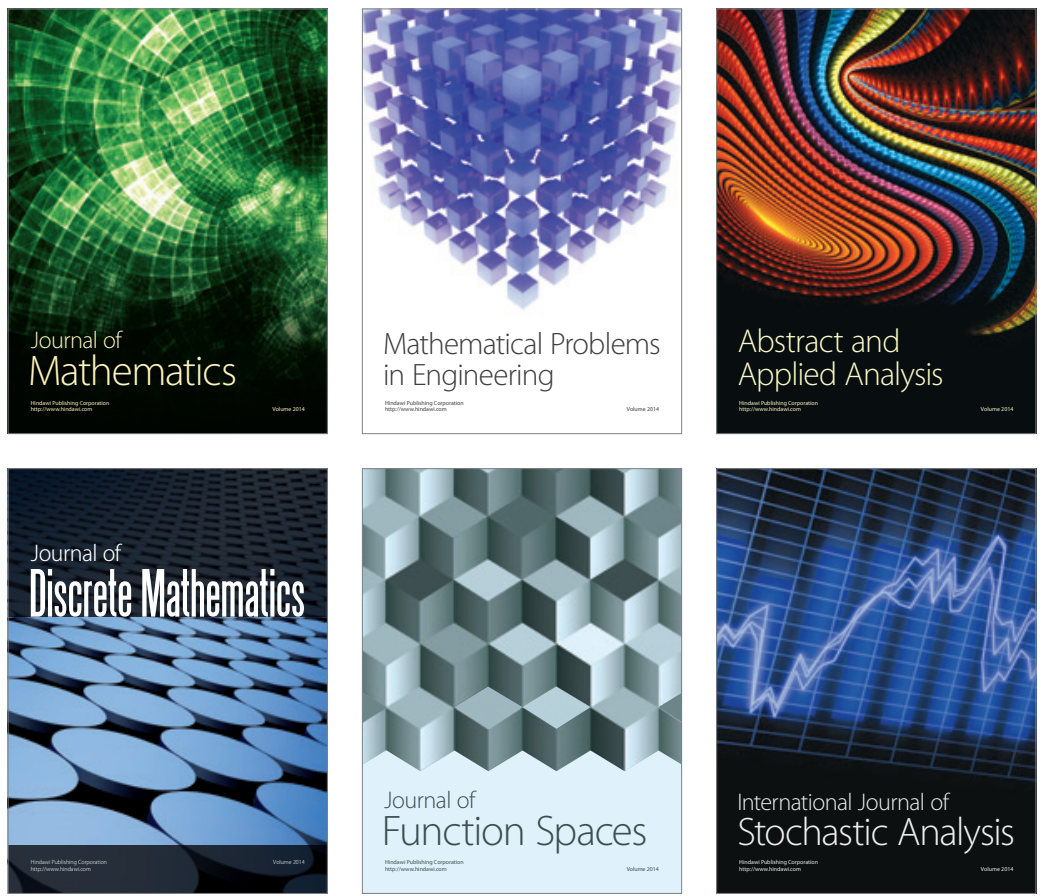

Journal of

Function Spaces

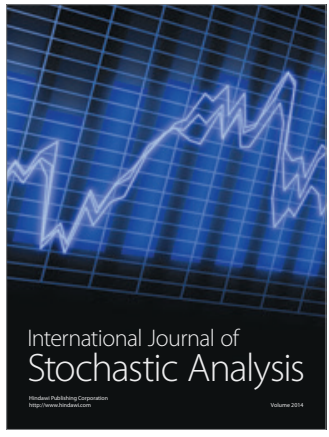

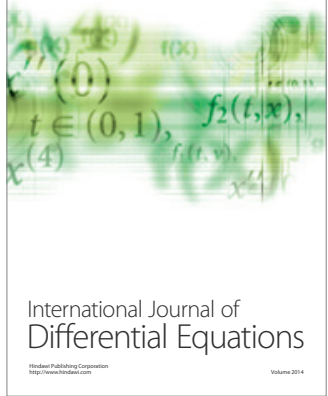
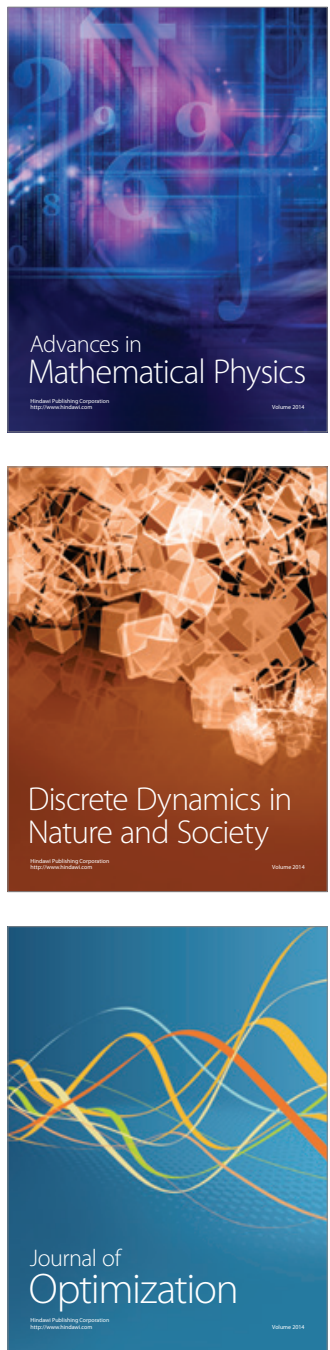